\title{
Memantine and Brain Atrophy in Alzheimer's Disease: A 1-Year Randomized Controlled Trial
}

\author{
David Wilkinson ${ }^{\mathrm{a}, *}$, Nick C. Fox ${ }^{\mathrm{b}}$, Frederik Barkhof ${ }^{\mathrm{c}}$, Ravinder Phul ${ }^{\mathrm{d}}$, Ole Lemming ${ }^{\mathrm{e}}$, \\ Philip Scheltens ${ }^{\mathrm{f}}$ and for the Study 10112 investigators \\ ${ }^{a}$ Memory Assessment and Research Centre, Moorgreen Hospital, Southampton, UK \\ ${ }^{\mathrm{b}}$ Dementia Research Centre, UCL Institute of Neurology, London, UK \\ ${ }^{\mathrm{c}}$ Department of Radiology, VU University Medical Center, MB, Amsterdam, The Netherlands \\ ${ }^{\mathrm{d}}$ Lundbeck Ltd UK, Milton Keynes, UK \\ ${ }^{\mathrm{e}} H$. Lundbeck A/S, Valby, Denmark \\ ${ }^{\mathrm{f}}$ Department of Neurology and Alzheimer Center, VU University Medical Center, Amsterdam, The Netherlands
}

Accepted 14 December 2011

\begin{abstract}
The primary objective of this exploratory study was to evaluate the rate of total brain atrophy (TBA) with serial magnetic resonance imaging (MRI), using the Brain Boundary Shift Integral (BBSI), in patients with probable Alzheimer's disease (AD) over the course of 52 weeks of treatment with memantine or placebo. This was a multi-national, randomized, double-blind, placebo-controlled, fixed-dose 1-year study. Patients were randomized $(1: 1)$ to treatment with placebo or memantine. Patients randomized to memantine were up-titrated to the target dose of $20 \mathrm{mg}$ /day over 4 weeks. MRI scans were collected at screening and at Weeks 4, 42, and 52. Secondary efficacy assessments included several cognitive and behavioral scales. 518 patients were screened, 278 patients were randomized, and 217 patients completed the study. In the primary efficacy analysis, the differences in TBA rates between memantine $(15.2 \mathrm{~mL} /$ year $)$ and placebo $(15.3 \mathrm{~mL} /$ year $)$ were not statistically significant $(-0.04 \mathrm{~mL} /$ year [(95\% CI: $-2.60,2.52), p=0.98])$. There was a statistically significant correlation between change in TBA and change in most cognitive and behavioral scale scores. Patients who were not treated with acetyl cholinesterase inhibitors (AChEIs) showed a significantly lower TBA rate than patients treated with AChEIs. Memantine had a placebo-level incidence of adverse events. There were no statistically significant differences between memantine and placebo in total brain or hippocampal atrophy rates in patients with probable AD treated for 1 year. The biological relevance of cerebral atrophy was supported by a significant correlation between rate of atrophy and decline in cognitive and behavioral outcomes.
\end{abstract}

Keywords: Acetyl cholinesterase inhibitors, behavior, cognition, memantine, MRI, placebo, total brain atrophy

\section{INTRODUCTION}

Two small pilot studies have shown effects of memantine on magnetic resonance imaging (MRI) parameters $[1,2]$. This exploratory study was designed

${ }^{*}$ Correspondence to: D. Wilkinson, Memory Assessment and Research Centre, Moorgreen Hospital, Southampton, SO3 3JB, United Kingdom. Tel.: +44 238047 5216; Fax: +44 238046 3022; E-mail: David.Wilkinson@southernhealth.nhs.uk. to evaluate the effect of memantine on the rate of total brain atrophy. Part of the rationale was to test the utility of a biomarker (MRI) as a primary outcome in a randomized controlled trial, as calculations suggested that an adequate power to detect change could be achieved on a much smaller sample size than would traditionally be required using clinical endpoints.

This exploratory study employed a novel design in a selected patient population, with an adequate period of observation (1 year) involving four MRI scans with 
appropriate scan intervals to enhance the precision in estimating the rate of brain atrophy [3]. Patients were chosen on the basis of being likely to tolerate MRI scans over the course of 1 year and likely to complete the study. Patients were included that were with or without treatment with an acetyl cholinesterase inhibitor (AChEI) (donepezil, rivastigmine, or galantamine), thereby conforming to the current standard of care.

The primary objective of this study was to explore the potential effects of memantine compared to placebo, on the rate of total brain atrophy in patients with Alzheimer's disease (AD) over 1 year using a validated serial MRI registration technique [4]. Treatment effects were stratified according to the AChEI status of the patients. Secondary objectives were to assess the effects of memantine on other MRI measures, that is, hippocampal volume change (atrophy). Other pre-defined secondary objectives were to evaluate the effects of memantine on cognitive and behavioral outcomes over 1 year, the potential associations between cognitive changes and MRI findings, and to assess the safety and tolerability of memantine over 1 year.

\section{METHODS}

\section{Design}

This multi-national, randomized, double-blind, parallel-group, placebo-controlled, fixed-dose study (http://www.clinicaltrials.gov/ct2/show/NCT008629 40) included 31 centers from 4 countries (France, Germany, Switzerland, and the United Kingdom). Outpatients with $\mathrm{AD}$ were recruited from specialist clinics from September 2005 to February 2009. The study was conducted according to the principles of Good Clinical Practice [5] and the Declaration of Helsinki [6]. Local ethics committees approved the study design and eligible patients gave their written informed consent before participating. Eligible patients were randomized in a $1: 1$ ratio to double-blind treatment with placebo or memantine for 52 weeks, stratified according to current AChEI treatment. Patients randomized to memantine started with a dose of $5 \mathrm{mg} /$ day and were up-titrated by $5 \mathrm{mg} /$ day once a week to the target dose of $20 \mathrm{mg} /$ day during a 4-week dose-escalation period. Once the target dose was reached, it remained fixed until the end of the 52-week period (Fig. 1). Medication was supplied in wallet cards, identified by visit, and patients (with the support of their caregiver) were instructed to take one tablet in the morning and one

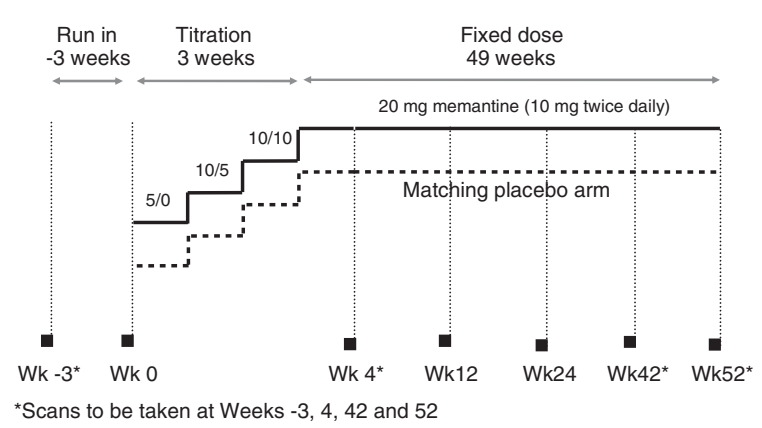

Fig. 1. Study design.

in the evening to maintain double blinding during titration and treatment periods.

\section{Patient population and enrichment procedure}

\section{Inclusion criteria}

Outpatients included in the study were $\geq 50$ years of age with a diagnosis of probable $\mathrm{AD}$, according to NINCDS-ADRDA criteria [7], and consistent with the MRI scan at screening. The patient needed to have a Mini Mental State Examination (MMSE) score $\geq 12$ and $\leq 20$ at screening and baseline [8], the patient should be otherwise healthy, ambulatory or ambulatory aided, with a reliable caregiver, and be a fluent speaker of the native language of the country. Women had to be at least 2 years post-menopausal or surgically sterile. Finally, patients with or without stable current AChEI treatment were allowed to be included in the study.

\section{Exclusion criteria}

Patients were excluded if there was evidence of clinically significant and active pulmonary, gastrointestinal, renal, hepatic, endocrine, or cardiovascular system disease, uncompensated congestive heart failure, or other organic disease. Patients with severe renal impairment, high or low blood pressure, hypersensitivity to memantine, amantadine, rimantidine or lactose, any clinically significant neurodegenerative disease or other serious neurological disorder other than $\mathrm{AD}$ were also excluded. Patients were excluded if they were unable to tolerate an MRI scan at screening or, in the opinion of the investigator, further scans scheduled during the study, or the patient was otherwise contraindicated for MRI. Patients with a Modified Hachinski Ischemia [9] score $>4$ at screening, or were foreseen to enter a nursing or residential home within the next 12 months were excluded from the study. 


\section{MRI inclusion and exclusion criteria (visual ratings)}

If the patient was considered inappropriate following centralized reading of his/her screening MRI scan, he/she was excluded. All screening MRI scans were visually rated by the VU University Medical Center, Amsterdam to further differentiate AD from other pathologies, and to exclude patients with vascular or non-Alzheimer neurodegeneration. Visual rating scales used were: the age-related white matter change (ARWMC) scale [10], with a score ranging between 0 and 30 for 5 different regions in the left and right hemispheres; global cortical atrophy (GCA) was scored using a 4-point rating scale (range 0-3), in which a score of 0 represents no atrophy, and a score of 3 severe atrophy [11]; medial temporal lobe atrophy (MTA) was rated using a five-point rating scale [12] ranging from 0 (no atrophy) to 4 (end stage atrophy) for left and right medial temporal lobe. A minimal MTA score of 1 on both sides (for patients $<65$ years) and at least 2 on one side and 1 on the other (for patients $\geq 65$ years) was required for inclusion.

Patients fulfilling the radiological criteria of the NINDS-AIREN (National Institute of Neurological Disorders and Stroke and Association Internationale pour la Recherche et l'Enseignement en Neurosciences) for vascular dementia [13], operationalized by van Straaten et al. [14] were excluded.

\section{Allocation to treatment}

The medication was given as tablets (memantine, 5 and $10 \mathrm{mg}$, and placebo) for oral administration. The placebo tablets were identical in appearance to the memantine tablets. Patients who fulfilled the selection criteria were assigned to 52 weeks of double-blind treatment in a $1: 1$ ratio to either placebo or memantine, in accordance with a computer-generated randomization list. The details of the randomization series were unknown to any of the investigators and were contained in a set of sealed opaque envelopes. At each study center, sequentially enrolled patients were assigned the lowest randomization number available in blocks of 4. To ensure that an equal number of patients within each category (that is, AChEI and non-AChEI treated patients) received memantine and placebo treatment, patients were randomized according to separate blocks. All study personnel and participants were blinded to treatment assignment for the duration of the entire study.

\section{Power and sample size calculations}

A minimum of 150 patients randomized to each treatment group (the all-patients randomized set, APRS) was estimated to be required to provide a power of at least $80 \%$ to detect a $20 \%$ slowing of the annual total brain atrophy rate, as a treatment effect of memantine at the 5\% two-sided significance level. This calculation was based on an expected between patient standard deviation in atrophy rate of $0.98 \%$ per year, a within-patient standard deviation in change of $0.82 \%$, and a brain boundary shift integral (BBSI)-related standard deviation of $0.05 \%$, and assumes that $90 \%$ of the patients reach Week $4,75 \%$ of the patients reach Week 42 and $70 \%$ reach Week 52.

\section{MRI scans}

A T1- and T2-weighted MRI scan was taken at the Screening Visit for visual ratings and baseline quantification of total brain and hippocampal volumes. Further T1-weighted MRI scans were conducted for quantification of changes in brain volume at Weeks 4, 42, and 52. MRI scans were conducted using 1.5 Tesla machines at qualified scanning centers and independently inspected by a central neuroimaging center (BioImaging, Leiden) for quality control. The scanning protocol at screening ( 3 weeks prior to randomization) included: 1) a 3D T1-weighted sequence: coronal orientation, whole brain coverage with $1.5 \mathrm{~mm}$ slice thickness; 2) a transverse fluid attenuated inversion recovery (FLAIR) sequence, $5 \mathrm{~mm}$ slice thickness, slice gap $0.5 \mathrm{~mm}$; 3) a transverse T2-weighted turbo spin echo sequence, $5 \mathrm{~mm}$ slice thickness, slice gap $0.5 \mathrm{~mm}$. All images were acquired with an in-plane resolution of $1 \times 1 \mathrm{~mm}$. The scanning protocol at all subsequent time-points following initiation of treatment (and used for quantification of changes in brain volume at Visits 3, 6, and 7) included only the 3D T1-weighted coronal sequence [15].

\section{Serial MRI efficacy measures}

Total brain direct measure of change was calculated using BBSI, which determines volume change over the entire three-dimensional brain-cerebrospinal fluid interface using a semi-automated iterative morphologic method by a central image analyses group (DRC, London). The technique has been described in detail [16]. In summary, the whole-brain region was first extracted from skull, scalp, and other soft tissue using a semi-automated technique [17]. The brain region was then checked and manually edited where necessary, and from this, an approximate measure of 
whole-brain volume was calculated. Each scan was then accurately co-registered (positionally matched) to its pair using a 9-df registration technique, after which the BBSI was calculated.

For hippocampal volume (HCV) measurement, all scans were first registered to a standard brain template using a 6-degrees-of-freedom algorithm to reduce variability in landmarks used in delineating the hippocampus by a central image analyses group (Image Analysis Centre, VU Medical Centre, Amsterdam). Repeat scans were then registered to corresponding baseline scans using a 9-degrees-of-freedom algorithm. Registered scan pairs were displayed simultaneously to improve consistency. Each hippocampus was manually traced using multiple views to include the cornu amonis, gyrus dentatus and subiculum [18]. Changes in right, left, and total (right + left) HCV was calculated by subtracting repeat from baseline HCVs.

\section{Cognitive and behavioral efficacy measures}

The following cognitive tests were used: Controlled Oral Word Association Test (COWAT); the Category Fluency Test (CFT) [19], the Stroop Interference Test (SIT), both congruent and incongruent [20], Orientation Test (OT) [21] and the Mini Mental State Examination (MMSE) [8]. Behavioral disturbances were assessed with the Neuropsychiatric Inventory (NPI) [22].

\section{Statistical analysis}

Safety analyses were based on the all-patientstreated set (APTS), which consisted of all randomized patients who took at least one dose of investigational medicinal product. Analyses involving cognitive and behavioral measures used the full-analysis set (FAScog), comprising all patients in the APTS who had at least one valid post-baseline efficacy measure. All analyses involving MRI measures (total brain atrophy and hippocampal atrophy) were conducted on the fullanalysis set MRI (FAS-MRI), which consisted of all patients in the APTS who had a valid MRI scan $\geq 6$ months after initiation of treatment.

\section{Primary efficacy analysis}

The primary efficacy analysis (estimates of treatment difference in total brain atrophy rate) was based on a linear mixed model relating direct change in brain volume (measured using BBSI) to time and its interaction with treatment group. Further aspects of the primary analysis involved use of the BBSI measures to additionally determine the rate of total brain volume (TBV) loss (brain atrophy). The model also included a time-by-AChEI group interaction as a fixed effect. The model also included a three-way interaction between treatment, AChEI, and time [23].

\section{Secondary efficacy analysis (MRI)}

The secondary efficacy analysis (estimates of treatment difference in hippocampal atrophy rate) was performed using Mixed Model Repeated Measurements (MMRM) with unstructured covariance including time, time-by-treatment interaction, visit, pre-treatment $\mathrm{HCV}$ interacting with visit, and AChEI group interacting with time.

\section{Secondary efficacy analyses (cognitive and behavioral)}

The secondary cognitive and behavioral outcomes were analyzed using MMRM and analysis of covariance (ANCOVA), analyzing the change from baseline. Treatment, AChEI status, and center were included as factors and the baseline total score (interacting with visit in the MMRM analyses) as covariate. All cognitive and behavioral variables were conducted on the FAS-cog using both the observed cases (OC) and the last observation carried forward (LOCF) approaches.

\section{Association analyses}

Association analyses between changes in cognitive/behavioral and MRI outcomes were conducted by including total brain atrophy or hippocampal atrophy, both separately and together, as predictors in the models analyzing cognitive and behavioral outcomes.

\section{Post-hoc analyses}

Annualized total brain and hippocampal atrophy rates were calculated from the change in total brain and hippocampal volumes from screening (week -3) to week 52, normalized to 52 weeks, expressed as a percentage of total brain and hippocampal volume at screening.

\section{Tolerability assessments}

Tolerability and safety were based on the incidence of adverse events, either reported spontaneously by the patients or in response to a non-leading question by the investigator throughout the study, vital signs (seated systolic/diastolic blood pressure and pulse rate), and weight. Safety data were collected at screening (except AEs), baseline, Weeks 4, 12, 24, 42, and 52, and weight 
was assessed at screening, baseline, Weeks 4, 42, and 52.

\section{RESULTS}

\section{Patient baseline characteristics}

Of the original 518 patients screened, almost half (47\%, $n=240$ ) were screening failures (Fig. 2). Reasons for screening failure (as a percentage of the number of patients screened), in decreasing incidence, were: unable to tolerate an MRI scan (11\%), insufficient evidence of AD according to MTA rating of the MRI scan (11\%), MRI evidence of vascular dementia according to radiological NINDS-AIREN criteria (8\%), MMSE score too high or too low (4\%), MRI evidence of other degenerative disorder $(3 \%)$, withdrawal of consent $(2 \%)$, other clinical criteria ( $8 \%$ ). The all-patients-treated set (APTS) comprised 277 patients (placebo: 144, memantine: 133). There were no clinically relevant differences between the two treatment groups in patient demographics (Table 1) or clinical characteristics at baseline (Table 2). Except for 1 patient, all were Caucasian, with a mean age of 74 years, a mean duration of illness of almost 4 years, and there were slightly more women than men. The FAS-cog consisted of 275 patients (placebo: 142, memantine: 133), with 198 patients taking AChEIs (placebo: 102, memantine: 96), and 77 patients not taking AChEIs (placebo: 40, memantine: 37). The FAS-MRI consisted of 228 patients (placebo: 118, memantine: 110), with 165 patients taking AChEIs (placebo: 86, memantine: 79), and 63 patients not taking AChEIs (placebo: 32, memantine: 31 ). The mean dose of AChEIs taken during the study (mg/day) was 9.2 (placebo) and 9.1 (memantine) for donepezil, 20.0 (placebo) and 21.6 (memantine) for galantamine, and 8.0 (placebo) and 8.7 (memantine) for rivastigmine.

\section{Withdrawals from the study}

A total of 60 patients $(22 \%)$ withdrew during the study, with 30 in each treatment group (Fig. 2). There was no difference between treatment groups in time to withdrawal (hazard ratio $=1.00, p=0.996$, Cox model). Approximately half of the withdrawals in each treatment group were due to adverse events (AEs). There were no statistically significant differ-

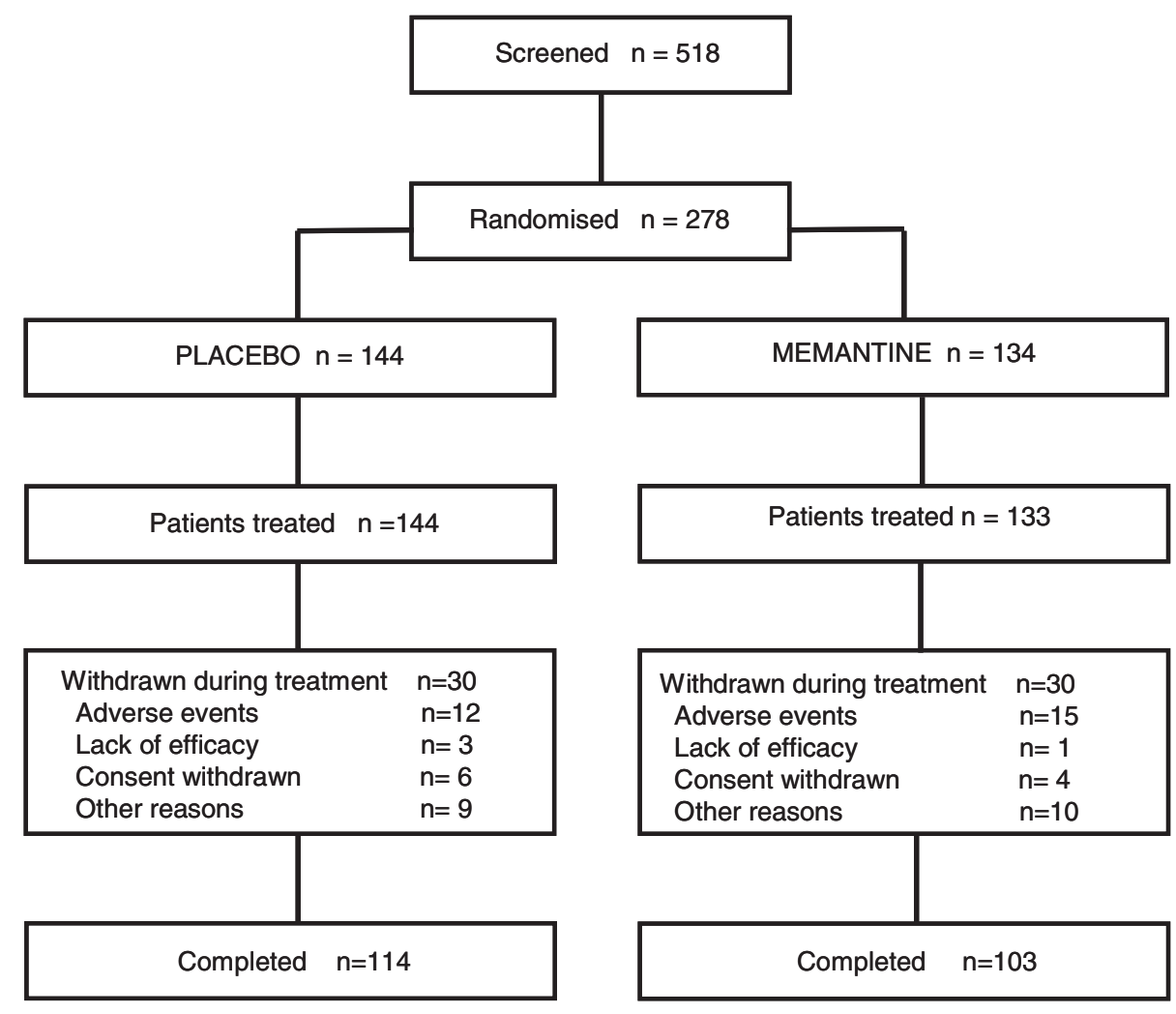

Fig. 2. CONSORT diagram of patient flow. 
Table 1

Patient demographics and baseline characteristics (APTS)

\begin{tabular}{lcc}
\hline & $\begin{array}{c}\text { Placebo } \\
(n=144)\end{array}$ & $\begin{array}{c}\text { Memantine } \\
(n=133)\end{array}$ \\
\hline Age; mean $( \pm \mathrm{SD})$ & $74(8)$ & $74(9)$ \\
Gender; $n(\%)$ women & $75(52 \%)$ & $83(62 \%)$ \\
Weight in kg $( \pm \mathrm{SD})$ & $71(14)$ & $69(14)$ \\
BMI in kg/m $( \pm \mathrm{SD})$ & $25.5(4)$ & $25.1(4)$ \\
Duration of disease; mean in years & 3.7 & 3.9 \\
Concurrent illnesses & & \\
Patients with ongoing history; $n(\%)$ & $124(86 \%)$ & $116(87 \%)$ \\
Hypertension & $58(40 \%)$ & $50(38 \%)$ \\
Hypercholesterolaemia & $21(15 \%)$ & $18(14 \%)$ \\
Osteoarthritis & $19(13 \%)$ & $18(14 \%)$ \\
Type 2 diabetes mellitus & $13(9 \%)$ & $11(8 \%)$ \\
Hypothyroidism & $13(9 \%)$ & $7(5 \%)$ \\
Depression & $10(7 \%)$ & $9(7 \%)$ \\
Asthma & $9(6 \%)$ & $4(3 \%)$ \\
Dyspepsia & $3(2 \%)$ & $7(5 \%)$ \\
\hline APTS: all-pation
\end{tabular}

APTS: all-patients-treated set, BMI: body mass index, SD: standard deviation.

Table 2

Screening variables

\begin{tabular}{lcc}
\hline & $\begin{array}{c}\text { Placebo } \\
(n=144)\end{array}$ & $\begin{array}{c}\text { Memantine } \\
(n=133)\end{array}$ \\
\hline MRI variables & & \\
TBV; mean (SD) (mL) & $968.9(108.3)$ & $957.9(103.3)$ \\
HCV; mean (SD) $\left(\mathrm{mm}^{3}\right)$ & $5107.6(836.7)$ & $5063.0(1014.3)$ \\
MTA; mean (SD) & $2.2(0.7)$ & $2.2(0.8)$ \\
ARWMC; mean (SD) & $4.2(3.3)$ & $4.4(3.7)$ \\
GCA; mean (SD) & $1.3(0.8)$ & $1.2(0.8)$ \\
Hachinski score; mean $( \pm S D)$ & $0.63(0.7)$ & $0.74(0.8)$
\end{tabular}

ARWMC: age-related white matter change, GCA: global cortical atrophy, HCV: hippocampal volume, MTA: medial temporal lobe atrophy, TBV: total brain volume.

ences between treatment groups in total withdrawals ( $p=0.73$, chi-squared test), due to AEs ( $p=0.41$, chisquared test), or due to lack of efficacy $(p=0.62$, Fisher's Exact test). The randomisation code was broken for 2 patients: 1 for an AE and only data collected from this patient until the time of code break were kept in the analysis sets. The code for the second patient was broken by mistake after study completion, and since this code break had no impact on the blinding of either the patient or the investigator during the study, the patient's data were kept in the study.

\section{Efficacy}

\section{Primary endpoint}

Based on the statistical model for the primary analysis of efficacy (estimates of treatment difference in total brain atrophy rate; FAS-MRI, OC), the difference of $-0.04 \mathrm{~mL} /$ year [(95\% CI: $-2.60,2.52), p=0.98]$ in favor of memantine was not statistically significant. Further aspects of the primary analysis revealed that the atrophy rates were $15.3(0.91)$ for placebo and 15.2 (0.97) for memantine [values are means (SE) in $\mathrm{mL} /$ year] (Table 3). Post-hoc analyses revealed that the annualized total brain atrophy rates [as a $\%$ of baseline volume (SD)] were 1.63 (1.05) for placebo and 1.48 (0.96) for memantine (Fig. 3A).

In the memantine group, the total brain atrophy rate was 16.6 (1.12) for patients using AChEIs compared to 11.9 (1.78) for patients not using AChEIs. In the placebo group, the total brain atrophy rate was 16.7 (1.05) for patients using AChEIs compared to 11.8 (1.72) for patients not using AChEIs [values are means (SE) in mL/year] (Table 3). For all patients, the difference in total brain atrophy rates for patients using AChEIs was 4.8 mL/year [(95\% CI: 1.92, 7.64), $p=0.001$ ], with significantly lower atrophy rates found in patients not treated with AChEIs. At screening, patients not on AChEI treatment had had a diagnosis of AD for approximately 1.4 years shorter than patients on AChEI treatment, but had no other clinically relevant differences.

The potential influence of other variables on the total brain atrophy rate was analyzed by separately adjusting for gender, disease duration, MRI center, MTA, ARWMC, GCA, country, baseline weight, baseline BMI, age, and baseline MMSE. Also added to the model were three-way interactions with gender, AChEI treatment duration, and duration of disease. No statistically significant interaction was shown between any of these variables and treatment effect. Sensitivity analyses, in which total brain atrophy was adjusted for baseline TBV, performed from Week 4, including only completers, did not change the overall conclusion that there was no statistically significant difference in total brain atrophy between the memantine and placebo groups.

\section{Secondary endpoints}

Hippocampal atrophy: Post-hoc analyses estimated that the hippocampal atrophy rate was 207 (174) $\mathrm{mm}^{3}[=4.2 \%$ (3.6) per year in the memantine group) and 207 (162) $\mathrm{mm}^{3}[=4.0 \%$ (3.2) per year in the placebo group] [values are means (SD)]. Based on the statistical model for the secondary analysis of efficacy (estimates of treatment difference in hippocampal atrophy rate; FAS-MRI, OC), it was found that relative to placebo, treatment with memantine yielded a nonsignificant difference of $3.5 \mathrm{~mm}^{3} /$ year in hippocampal atrophy rate $(p=0.84)$. Relative to not using AChEIs, treatment with AChEIs yielded a non-significant 
Table 3

Brain atrophy rates based on BBSI measurements (mL/year \pm SD) (FAS-MRI, OC)

\begin{tabular}{llrr}
\hline & Placebo & Memantine & $\begin{array}{r}\text { Treatment difference (95\% CI) } \\
\text { difference }(95 \% \text { CI) }\end{array}$ \\
\hline All patients & $15.3 \pm 9.9(n=118)$ & $15.2 \pm 10.2(n=110)$ & $-0.04(-2.60 \text { to } 2.52)^{\mathrm{a}}$ \\
AChEI use: yes & $16.7 \pm 11.4(n=86)^{\mathrm{b}}$ & $16.6 \pm 11.7(n=79)^{\mathrm{c}}$ & - \\
AChEI use: no & $11.8 \pm 18.7(n=32)$ & $11.9 \pm 18.7(n=31)^{\mathrm{d}}$ & $4.78(1.92 \text { to } 7.64)^{\mathrm{e}}$ \\
Annualized total brain atrophy rate $\left(\%\right.$ per year) ${ }^{\mathrm{f}}$ & $1.63 \pm 1.05(n=109)$ & $1.48 \pm 0.96(n=88)$ & $-0.14 \pm 1.0^{\mathrm{f}}$ \\
\hline
\end{tabular}

${ }^{\mathrm{a}} p=0.975$ memantine versus placebo; ${ }^{\mathrm{b}} p=0.016$ versus placebo, no AChEI use; ${ }^{\mathrm{c}} p=0.020$ versus placebo, no AChEI use; ${ }^{\mathrm{d}} p=0.966$ versus placebo, no AChEI use; ${ }^{\mathrm{e}} p=0.001$ AChEI use $(n=165)$ versus no AChEI use $(n=63)$; ${ }^{\mathrm{f}} p=0.268$ memantine versus placebo BBSI: Brain Boundary Shift Integral, CI: confidence interval.
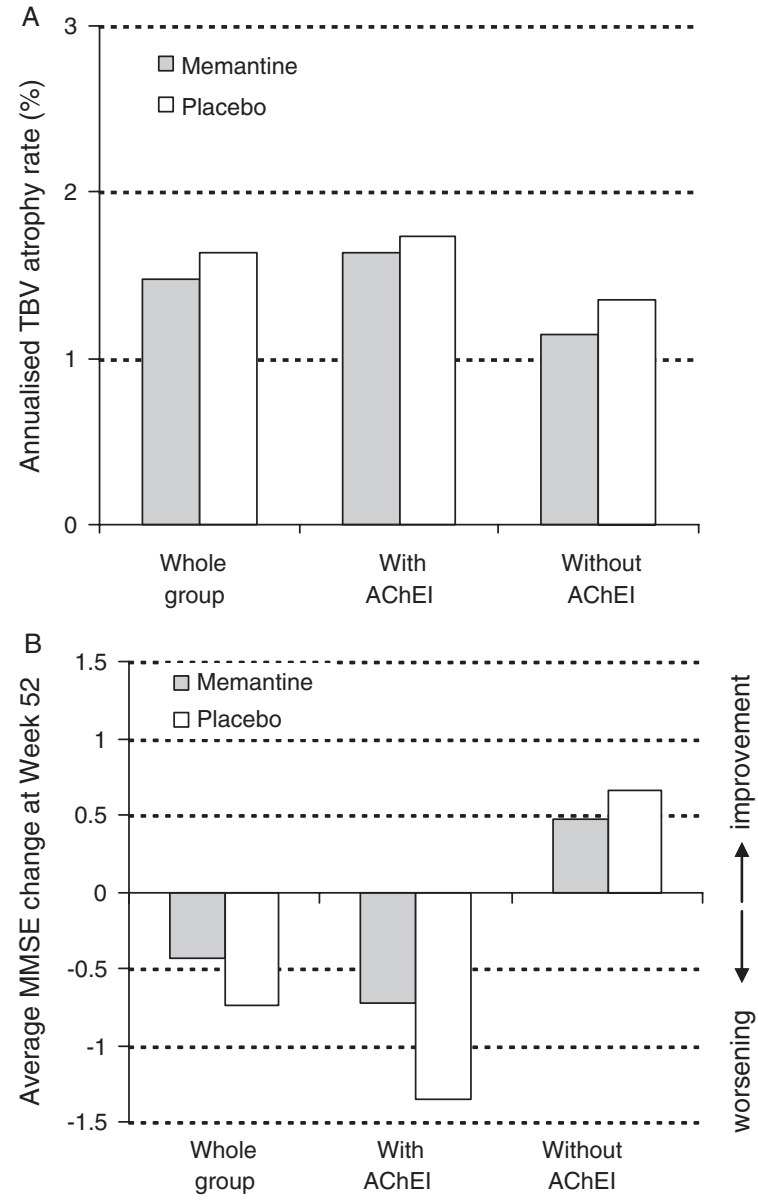

Fig. 3. A) Annualized total brain volume (TBV) atrophy rates for placebo and memantine, for all patients, and by acetyl cholinesterase inhibitor (AChEI) subgroup. B) Mean change in MMSE score from baseline to Week 52 for all patients, and by AChEI subgroup (FAS, OC).

difference in hippocampal atrophy rate of $2.9 \mathrm{~mm}^{3} /$ year $(p=0.88)$.

Cognitive and behavioral outcomes: Memantinetreated patients improved statistically significantly more on the COWAT at Weeks 12, 24, 42, and 52
(MMRM) and on the CFT at Weeks 24 and 52 (MMRM) than did placebo-treated patients (FAS-cog). There were no statistically significant differences in changes from baseline on the OT, SIT-C, SIT-I, MMSE, or NPI total scores between memantine- and placebotreated patients at any time point. On the MMSE, the mean annual change (SE) was $-0.74(0.48)$ for placebo and -0.43 (0.49) for memantine (FAS-MRI, OC). In the placebo subgroup without AChEIs, there was an improvement from baseline in MMSE scores of 0.66 (0.66) over 52 weeks compared to placebo patients with AChEIs, who showed a worsening of $-1.35(0.49)$ (FAS-cog, OC, ANCOVA) (Fig. 3B). When looking at performance in the placebo group for the other scales, in comparison to patients on AChEIs, patients who were not receiving AChEI treatment showed improvements on the NPI, less decline from baseline in CFT and Stroop-C total scores, but inconsistent changes from baseline on COWAT, Orientation and Stroop-I total scores (Table 4).

Association analyses: There was a statistically significant correlation (ranging from 0.16 to 0.42 ) between change in total brain atrophy over 1 year and change in all cognitive and behavioral scale scores (except SITI), with increase in total brain atrophy being related to deterioration in scale scores. No significant correlation was found between the scale scores and hippocampal atrophy (Table 5).

\section{Safety}

Three patients in the memantine group and 1 patient in the placebo group died during the study; one from cancer, one from intracerebral hemorrhage, one following a car accident, and one from circulatory failure. The incidence of serious adverse events (SAEs), including those with fatal outcome, was similar in the memantine (13\%) and placebo (14\%) groups. In both treatment groups, fall was the SAE with the highest incidence (memantine: 4 patients; placebo: 8 patients). 
Table 4

Mean $( \pm \mathrm{SD})$ efficacy scores (FAS)

\begin{tabular}{|c|c|c|c|c|c|}
\hline & \multicolumn{2}{|c|}{ Baseline } & \multicolumn{3}{|c|}{ Mean change (SE) from baseline to Week 52: difference to placebo } \\
\hline & $\begin{array}{l}\text { Placebo } \\
(n=144)\end{array}$ & $\begin{array}{c}\text { Memantine } \\
(n=133)\end{array}$ & $\mathrm{OC}$ & LOCF & MMRM \\
\hline COWAT & $19.2(8.8)$ & $19.7(10.3)$ & $1.40(0.92)$ & $1.41(0.82)$ & $1.68(0.79)^{*}$ \\
\hline CFT & $13.3(5.9)$ & $13.5(5.8)$ & $1.07(0.61)$ & $0.82(0.53)$ & $1.37(0.52)^{* *}$ \\
\hline ADAS-cog-OT & $4.0(1.7)$ & $4.2(1.6)$ & $-0.08(0.23)$ & $-0.13(0.20)$ & $-0.05(0.20)$ \\
\hline Stroop C & $68.8(41.7)$ & $70.5(49.1)$ & $-1.19(6.69)$ & $-0.58(5.77)$ & $-3.10(4.87)$ \\
\hline Stroop-I & $176.4(56.7)$ & $170.3(60.4)$ & $5.76(6.86)$ & $9.86(5.80)$ & $7.54(5.57)$ \\
\hline MMSE & $17.1(2.4)$ & $16.7(2.4)$ & $0.14(0.48)$ & $0.19(0.42)$ & $0.24(0.46)$ \\
\hline NPI & $12.8(12.4)$ & $13.1(12.8)$ & $0.68(1.42)$ & $0.61(1.29)$ & $0.56(1.19)$ \\
\hline
\end{tabular}

COWAT: controlled oral word association test, CFT: category fluency test, ADAS-cog-OT: Alzheimer's disease assessment scale - cognitive subscore - orientation test, MMSE: Mini-Mental State Examination, NPI: neuropsychiatric inventory. OC: observed cases, LOCF: last observation carried forward, MMRM: mixed model, repeated measures. $* p<0.05, * * p<0.01$.

Table 5

Association analyses between scale scores and MRI measurements (FAS-MRI, OC)

\begin{tabular}{|c|c|c|c|c|c|c|c|}
\hline & COWAT & CFT & $\begin{array}{l}\text { ADAS- } \\
\text { cog-OT }\end{array}$ & SIT-C & SIT-I & MMSE & NPI \\
\hline Brain atrophy rate ${ }^{\mathrm{a}}$ & $\begin{array}{c}-0.22(0.04) \\
r=-0.32 \\
p<0.001\end{array}$ & $\begin{array}{c}-0.16(0.03) \\
r=-0.28 \\
p<0.001\end{array}$ & $\begin{array}{l}0.05(0.01) \\
r=0.28 \\
p<0.001\end{array}$ & $\begin{array}{c}1.18(0.30) \\
r=0.22 \\
p<0.01\end{array}$ & $\begin{array}{c}1.17(0.36) \\
r=0.11 \\
p>0.05\end{array}$ & $\begin{array}{c}-0.13(0.02) \\
r=-0.42 \\
p<0.001\end{array}$ & $\begin{array}{c}0.15(0.06) \\
r=0.16 \\
p<0.05\end{array}$ \\
\hline Hippocampal atrophy rate ${ }^{\mathrm{b}}$ & $\begin{array}{c}0.00(0.00) \\
r=-0.04 \\
p<0.05\end{array}$ & $\begin{array}{l}0.00(0.00) \\
r=0.05 \\
p<0.05\end{array}$ & $\begin{array}{c}0.00(0.00) \\
r=-0.13 \\
p<0.05\end{array}$ & $\begin{array}{c}0.03(0.02) \\
r=0.03 \\
p<0.05\end{array}$ & $\begin{array}{c}0.04(0.02) \\
r=0.12 \\
p<0.05\end{array}$ & $\begin{array}{c}0.00(0.00) \\
r=0.03 \\
p<0.05\end{array}$ & $\begin{array}{c}0.00(0.00) \\
r=-0.04 \\
p<0.05\end{array}$ \\
\hline
\end{tabular}

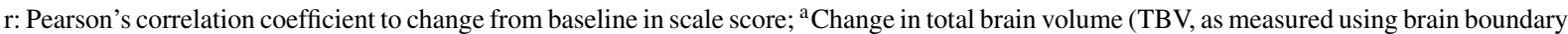
shift integral [BBSI], mL) between screening and Week 52; ${ }^{\mathrm{b}}$ Change in hippocampal volume (HCV, $\left.\mathrm{mm}^{3}\right)$ between screening and Week 52.

Slightly more than $50 \%$ of the patients had AEs, and the incidence was similar in each treatment group. The system organ classes (SOCs) with the highest incidences $(\geq 20 \%)$ of AEs were nervous system disorders (memantine: 23\%; placebo: 12\%) and psychiatric disorders (memantine: 22\%; placebo: $11 \%$ ). In both treatment groups, the $\mathrm{AE}$ with the highest incidence was fall; memantine: 9\%; placebo: $12 \%$. Only agitation had an incidence $\geq 5 \%$ and a statistically significantly higher incidence in the memantine group (5\%) than in the placebo group $(1 \%)$. None of the 7 patients in the memantine group with agitation withdrew from the study because of the AE.

The incidence of AEs considered related to the investigational medicinal product by the investigator was $32 \%$ in the memantine group and $22 \%$ in the placebo group. In the memantine group, 5 patients had severe, related adverse events (atrial fibrillation and cardiac failure [SAEs], visual acuity reduced, constipation, ankle fracture and fall [SAEs], delusion [SAE]). In the placebo group, 6 patients had severe, related adverse events (fall and femoral neck fracture [SAEs], convulsion [SAE], somnolence and urinary incontinence [SAEs], anxiety, hypotension [SAE], abnormal behavior).
The incidence of AEs that contributed to withdrawal was $11 \%$ in the memantine group and $8 \%$ in the placebo group. The AEs were distributed across many symptoms and diagnoses with no apparent trend. Except for headache (3 patients in the memantine group), no $\mathrm{AE}$ contributed to the withdrawal in $\geq 2$ patients in either treatment group.

No clinically relevant mean changes from baseline in vital signs were seen during the study. The mean weight change from baseline (screening) to Week 52 was $1.4 \mathrm{~kg}$ in the memantine group and $-0.1 \mathrm{~kg}$ in the placebo group. In the memantine group, the proportion of patients with a weight increase $>7 \%(22.5 \%)$ was higher than the proportion of patients with a weight decrease $<7 \%$ (7.5\%), while in the placebo group the proportion of patients with weight increase was similar to that of patients with weight decrease $(8.8 \%)$. No patients withdrew from the study due to weight changes.

\section{DISCUSSION}

This exploratory study had a novel design in which a biomarker was used as a primary outcome for the first time in an AD treatment study. Results indicated no 
effect of memantine on the rate of total brain atrophy. We found that both total brain atrophy rate and the MMSE decline were lower than previously reported. In the placebo group, the total brain atrophy rate was lower (1.6\% per year) than seen in some other studies (2.2\% per year) $[3,24]$, as was the MMSE decline (0.7 points versus 2.2 points per year) [25]. Interestingly, the rates of atrophy in this study were similar to those recently reported from the ADNI [26].

A great deal of effort was put into standardization of MRI scan acquisition, diagnoses and analyses to control variability associated with MRI data and to achieve precision in estimating rates of MRI change over time. Use of multiple time points improved precision in estimating rates of atrophy: the SDs of the annualized total brain atrophy rates were similar to that used to calculate sample size $(0.98 \%)$ based on single center MRI scans. Also, the MRI schedule allowed, in the event of a significant difference between groups, assessment of short term effects ( $0-4$ weeks) to distinguish immediate drug presence effects from sustained effects on rates of atrophy.

Standardization of diagnoses was also seen as important with a unique patient selection of an "enriched" population of patients. This was done to improve the specificity of the clinical diagnosis of $\mathrm{AD}$, to control for the heterogeneity in the patient population, and it was assumed it would increase the likelihood of seeing change over time in whole brain and hippocampal volume. This assumption was proved to be incorrect, but this study did show that this kind of standardization was achievable in this multi-center setting to the extent that the variability was in line with that demonstrated for single-site studies.

More than the usual caution should be exercised when interpreting the efficacy results of this study and in making generalizations to a broader AD population, as this obviously constitutes quite a selected sample. The patient population was different from previous trials $[1,27]$, chosen for the very reason that they would be stable (able to tolerate MRI scanning for a whole year) and homogenous (we used specific MRI criteria and enrichment) and this clearly may have implications for our results.

It is noteworthy that pre-selection of patients with evidence of MTA and minimal vascular changes did not select a group of patients who showed the typically expected radiological or clinical decline over one year, in contrast to the Henneman study [28]. The dissociation between MTA and the clinical decline in this study may suggest a dissonance between this biomarker and clinical progression.
That such a traditional hallmark of the disease may not relate to the clinical syndrome or progression as clearly as was thought, may mean we need something more than MTA or indeed MMSE score to identify those patients likely to progress and who would be most helpful to enroll into clinical trials. Furthermore, it could be that not excluding patients with small vessel disease may have influenced the overall decline, but this was not the case in the Henneman data [28].

An interesting finding was the difference between AChEI subgroups for total brain atrophy and MMSE decline. In fact $30 \%$ of the patients in this study, despite it being a year long and in moderate $\mathrm{AD}$, were not treated with AChEIs and yet showed a significantly lower total brain atrophy rate than patients treated with AChEIs. Furthermore, it was observed that the total brain atrophy rate in patients not treated with AChEIs was not typical of the overall patient population either.

Added to the fact that a lower rate of cognitive decline was seen in those patients who were not being treated with an AChEI, this may have implications for selection of patients for future trials in AD. This was a difficult study to recruit to and those recruited who were not on an AChEI were probably a rather abnormal group of stable patients, albeit with demonstrable memory complaints. Those on an AChEI were probably more typical of the AD patients we see clinically and it seems that these patients should form the basis of future trials with AD therapies. Those on AChEI may have also contained a higher proportion of patients with an APOE4 allele but unfortunately this was not measured in the study and represents another limitation of the study. If untreated AD patients are included, then the reasons they are not being treated need to be clearly recorded, as these reasons may influence their progression, making them unsuitable for inclusion in treatment trials.

\section{CONCLUSIONS}

In this study, we have shown that the enrichment of subjects did not increase the number of patients showing atrophy or clinical decline, perhaps suggesting that the presence of MTA itself was not a good indicator of future cognitive decline. The enriched population in this study suggests caution should be exercised when interpreting these results and in generalizing findings to a broader AD population. There was a statistically significant correlation between change in total brain atrophy over 1 year and change in all cognitive and behavioral scale scores (except SIT-I). 
These findings are in line with reports of good correlation between global measures of atrophy and cognition versus regional measures [3, 25]. An MRI marker may have limitations due to cost, as clinical measures are easier and cheaper to perform, but it adds an objective correlation with a link to the pathology that adds credence to any clinical study results. In addition, a number of AD patients, particularly when more severely affected, may not tolerate MRI scans. Although we could not show statistically significant effects on reducing brain atrophy with treatment in this study, we have shown that such a multi-center study can be undertaken with good accuracy, which has implications for future research. Memantine had a low incidence of adverse events.

\section{ACKNOWLEDGMENTS}

This study was sponsored by H. Lundbeck A/S and Merz Pharmaceuticals GmbH.

Authors' disclosures available online (http://www.jalz.com/disclosures/view.php?id=1098).

\section{REFERENCES}

[1] Schmidt R, Ropele S, Pendl B, Ofner P, Enzinger C, Schmidt H, Berghold A, Windisch M, Kolassa H, Fazekas F (2008) Longitudinal multimodal imaging in mild to moderate Alzheimer disease: A pilot study with memantine. J Neurol Neurosurg Psychiatry 79, 1312-1317.

[2] Weiner M, Graham SM, Hofbauer RK, Yu SY, Li S, Hsu H-A, Suhy J, Perhach JL (2008) Memantine treatment in patients with Alzheimer's disease is associated with a slower right hippocampal volume loss: An open-label, multi-center trial. Poster presented at the 11th International Conference on Alzheimer's Disease (ICAD); Chicago, IL, USA.

[3] Schott JM, Crutch SJ, Frost C, Warrington EK, Rossor MN, Fox NC (2008) Neuropsychological correlates of whole brain atrophy in Alzheimer's disease. Neuropsychologia 46, 17321737.

[4] Fox NC, Scahill RI, Crum WR, Rossor MN (1999) Correlation between rates of brain atrophy and cognitive decline in AD. Neurology 52, 1687-1689.

[5] ICH (1996) ICH Harmonised Tripartite Guideline E6: Guideline for Good Clinical Practice.

[6] World Medical, Association (WMA) (1964) Declaration of Helsinki: Ethical principles for medical research involving human subjects, http://www.wma.net/en/30publications/ 10policies/b3/

[7] McKhann G, Drachman D, Folstein M, Katzman R, Price D, Stadlan EM (1984) Clinical diagnosis of Alzheimer's disease: Report of the NINCDS-ADRDA work group under the auspices of department of health and human services task force on Alzheimer's Disease. Neurology 34, 939-944.

[8] Folstein MF, Folstein SE, McHugh PR (1975) "Mini-mental state." A practical method for grading the cognitive state of patients for the clinician. J Psychiatr Res 12, 189-198.
[9] Rosen WG, Terry RD, Fuld PA, Katzman R, Peck A (1980) Pathological verification of ischemic score in differentiation of dementias. Ann Neurol 7, 486-488.

[10] Wahlund LO, Barkhof F, Fazekas F, Bronge L, Augustin M, Sjögren M, Wallin A, Ader H, Leys D, Pantoni L, Pasquier F, Erkinjuntti T, Scheltens P, European. Task Force on AgeRelated White Matter Changes (2001) A new rating scale for age-related white matter changes applicable to MRI and CT. Stroke 32, 1318-1322.

[11] Pasquier F, Leys D, Weerts JG, Mounier-Vehier F, Barkhof F, Scheltens P (1996) Inter- and intraobserver reproducibility of cerebral atrophy assessment on MRI scans with hemispheric infarcts. Eur Neurol 36, 268-272.

[12] Scheltens P, Leys D, Barkhof F, Huglo D, Weinstein HC, Vermersch P, Kuiper M, Steinling M, Wolters EC, Valk J (1992) Atrophy of medial temporal lobes on MRI in "probable" Alzheimer's disease and normal ageing: Diagnostic value and neuropsychological correlates. J Neurol Neurosurg Psychiatry 55, 967-972.

[13] Román GC, Tatemichi TK, Erkinjuntti T, Cummings JL, Masdeu JC, Garcia JH, Amaducci L, Orgogozo JM, Brun A, Hofman A, Moody DM, O'Brien MD, Yamaguchi T, Grafman J, Drayer BP, Bennett DA, Fisher M, Ogata J, Kokmen E, Bermejo F, Wolf PA, Gorelick PB, Bick KL, Pajeau AK, Bell MA, DeCarli C, Culebras A, Korezyn AD, Bogousslavsky J, Hartman A, Scheinberg P (1993) Vascular dementia: Diagnostic criteria for research studies. Report of the NINDS-AIREN International Workshop. Neurology 43, 250-260.

[14] van Straaten EC, Scheltens P, Knol DL, van Buchem MA, van Dijk EJ, Hofman PA, Karas G, Kjartansson O, de Leeuw FE, Prins ND, Schmidt R, Visser MC, Weinstein HC, Barkhof F (2003) Operational definitions for the NINDS-AIREN criteria for vascular dementia: An interobserver study. Stroke 34, 1907-1912.

[15] Henneman WJ, Sluimer JD, Cordonnier C, Baak MM, Scheltens P, Barkhof F, van der Flier WM (2009) MRI biomarkers of vascular damage and atrophy predicting mortality in a memory clinic population. Stroke 40, 492-498.

[16] Freeborough PA, Fox NC (1997) The boundary shift integral: An accurate and robust measure of cerebral volume changes from registered repeat MRI. IEEE Trans Med Imag 16, 623629.

[17] Freeborough PA, Fox NC, Kitney RI (1997) Interactive algorithms for the segmentation and quantitation of 3-D MRI brain scans. Comput Methods Programs Biomed 53, 15-25.

[18] van de Pol LA, Barnes J, Scahill RI, Frost C, Lewis EB, Boyes RG, van Schijndel RA, Scheltens P, Fox NC, Barkhof F (2007) Improved reliability of hippocampal atrophy rate measurement in mild cognitive impairment using fluid registration. Neuroimage 34, 1036-1041.

[19] Wechsler D (1987) Wechsler Memory Scale-Revised manual. San Antonio, Texas, The Psychological Corporation.

[20] Jensen AR, Rohwer WD Jr (1966) The Stroop color-word test: A review. Acta Psychol (Amst) 25, 36-93.

[21] Rosen WG, Mohs RC, Davis KL (1984) A new rating scale for Alzheimer's disease. Am J Psychiatry 141, 1356-1364.

[22] Cummings JL (1997) The Neuropsychiatric Inventory: Assessing psychopathology in dementia patients. Neurology 48(5 Suppl 6), S10-S16.

[23] Frost C, Kenward MG, Fox NC (2004) The analysis of repeated 'direct' measures of change illustrated with an application in longitudinal imaging. Stat Med 23, 3275-3286.

[24] Ridha BH, Anderson VM, Barnes. J, Boyes RG, Price SL, Rossor MN, Whitwell JL, Jenkins L, Black RS, Grundman 
M, Fox NC (2008) Volumetric MRI and cognitive measures in Alzheimer disease: Comparison of markers of progression. J Neurol 255, 567-574.

[25] Jack CR Jr, Slomkowski M, Gracon S, Hoover TM, Felmlee JP, Stewart K, Xu Y, Shiung M, O'Brien PC, Cha R, Knopman D, Petersen RC (2003) MRI as a biomarker of disease progression in a therapeutic trial of milameline for AD. Neurology 60, 253-260.

[26] Evans MC, Barnes J, Nielsen C, Kim LG, Clegg SL, Blair M, Leung KK, Douiri A, Boyes RG, Ourselin S, Fox NC; Alzheimer's Disease Neuroimaging Initiative (2010) Volume changes in Alzheimer's disease and mild cognitive impairment: cognitive associations. Eur Radiol 20, 674-682.

[27] Krishnan KR, Charles HC, Doraiswamy PM, Mintzer J, Weisler R, Yu X, Perdomo C, Ieni JR, Rogers S (2003) Randomized, placebo-controlled trial of the effects of donepezil on neuronal markers and hippocampal volumes in Alzheimer's disease. Am J Psychiatry 160, 2003-2011.

[28] Henneman WJ, Sluimer JD, Cordonnier C, Baak MM, Scheltens P, Barkhof F, van der Flier WM (2009) MRI biomarkers of vascular damage and atrophy predicting mortality in a memory clinic population. Stroke 40, 492-498. 